\title{
Design and Implementation of a Real Time Wireless Monitor System for Urinary Incontinence
}

\author{
Xuhui Nie, Zongyu Song, Jinsong Yang, Ziwei Dengyun, Mingxi Yin, Xiaohui Duan, Bingli Jiao \\ Centre of Wireless Communication and Signal Processing, School of Electronics Engineering and Computer Science, Peking \\ University, Beijing, China \\ Email: 1401214246@pku.edu.cn, duan@pku.edu.cn
}

How to cite this paper: Nie, X.H., Song, Z.Y., Yang, J.S., Dengyun, Z.W., Yin, M.X., Duan, X.H. and Jiao, B.L.(2017) Design and Implementation of a Real Time Wireless Monitor System for Urinary Incontinence. Int. J. Communications, Network and System Sciences, 10, 252-263.

https://doi.org/10.4236/ijcns.2017.105B025

Received: May 1, 2017

Accepted: May 23, 2017

Published: May 26, 2017

\begin{abstract}
Urinary incontinence is the most common health problem in aged people. Leaving incontinence events unmanaged will make a negative influence on the aged and the patient both mentally and physically. This paper presents a design and implementation of a real time wireless monitor system for urinary incontinence, which has been applied in two nursing homes in Beijing and Shanghai. We collect real time moisture information by using non-contact humidity sensor designed and manufactured by us. When urinary incontinence, the sensor will send alert to mobile device via Bluetooth. If got the alert, the mobile device will send the alert to relatives of the aged or disabled people and cloud computing platform, through which we can make this information managed and displayed and the paramedic can be informed about the alarm. This paper gives a brief introduction to the framework of this system, the design of the non-contact sensor, the transmission of wireless data and the results of tests.
\end{abstract}

\section{Keywords}

Urinary Incontinence, Non-Contact Sensor, Real-Time, Apps, Cloud Computing Platform, Communication Protocol

\section{Introduction}

China has entered an aging society, the number of aging population increases rapidly, which will increase 5960 thousand each year from 2001 to 2020 [1]. According to the survey in hospitals and nursing homes about the demand of a monitoring system for the aged or disabled people, we find the importance of monitoring urinary incontinence. In the traditional way, the paramedics need to 
do a manual comprehensive exam for each few hours to check whether the diaper is wet or not. Wet diapers not changed for a long time will result in pressure sore, urinary tract infection, flamed skin and peculiar smell of the body. What's even worse is that it will bring negative feelings to the aged or disabled people. The artificial checking method also brings greater pressure to paramedics, especially in China, where is in a great lack of sufficiently qualified paramedics. Under this current circumstance, the real time monitoring of urinary incontinence will greatly decrease the response time for changing diapers after urinary incontinence so the aged or disabled people can get rapid attendance and the paramedics will release from the heavy and duplicate work.

Existing devices and products have already achieved non-contact urinary incontinence monitoring, based on capacitance sensor and transmission of moisture information through the wireless network [2]. Some other products, based on built-in moisture sensor and RF transmitter, triggered and send alarm by monitoring the change of humidity [3] [4]. And some can monitor the sensor of Urinary incontinence and send the alarm to wireless gateway, then send to the host PC [5]. But they have some limits in some aspect; some of them cannot realize non-contact sensing, long-distance transmission or have problem in sending and processing the urinary incontinence alarm information.

This paper introduces a real time wireless monitor system designed by us for urinary incontinence of aged or disabled people in order to improve the quality of attendance and reduce the pressure of paramedics. The main challenges we face are non-contact humidity measuring, reliable transmission and handling of urinary incontinence alerts. Based on non-contact resistance humidity sensor which also supports shedding detection, we do many tests and collect these data in order to determine the best threshold of urine moisture alarm. In the way of transmission of urine moisture alarm, we designed a bidirectional protocol for Bluetooth communication between sensor and mobile device and the wireless communication between mobile device and cloud computing platform to ensure the reliable and timely transmission of alert information. The cloud computing platform will store and process urinary incontinence information and make paramedics informed of the alert by sending messages and displaying alert on the website. All the data stored in the cloud will provide a basis for big data analysis.

The rest of the paper is organized as follows: Section 2 is an overall introduction of the system and the method of the system design. Section 3 is the introduction of each module of the system: communication process, the design of the humidity sensor, software design and hardware design of the acquisition and transmission device, the design of the app on mobile device, design of cloud computing platform and communication protocol between them. Section 4 is the material object of the system and the testing result. Section 5 is the abstract of the design and some potential improvement in the practical application.

\section{System Overview}

Figure 1 shows an overview of the system, which includes humidity sensor, ac- 


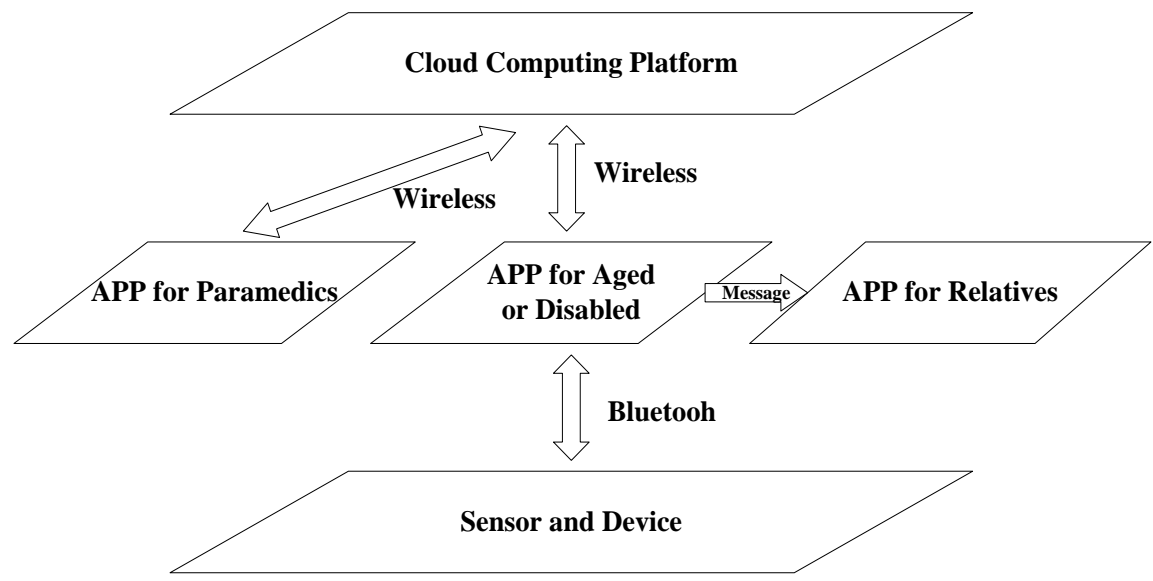

Figure 1. Overview of the monitor system.

quisition and transmission device, mobile device and cloud computing platform.

\subsection{Humidity Sensor and Embedded Device}

We designed and manufactured the humidity sensor, which will be put under a diaper, based on measuring the change in resistance value. The humidity sensor is non-contact and thin, so it won't bring any foreign body sensation to the aged or disabled people. As a result, they will not feel uncomfortable and the sensor will not do harm to the skin.

Acquisition and transmission embedded device must be reliable and low-power, at the same time, have some useful function like torch and manual alarm. Due to the requirement of long time continuous monitoring and complicated Bluetooth communication, real time operating system is necessary for embedded system. The acquisition and transmission device is connected with the sensor in the wired way so that we can replace the sensor easily, as a result of which we can realize long time monitoring of the aged or disabled people.

\subsection{Mobile Device}

Mobile devices of the system are developed based on Android equipment. These devices are equipped with Bluetooth hardware so that they can communicate with the embedded device. Software on the mobile device has two parts: the software for the aged or disabled people and the software for the paramedic. The software for the aged or disabled people is used to communicate with the embedded device, system configuration of the device and acquisition of alert information and system information from the device. Also, the software needs to communicate with cloud server, register and manage devices, uploading alert information and sending alert message to relatives in order to realize the multiple reliable release of the alert. The software for the paramedic needs to communicate with cloud computing platform, getting the information of the aged or disabled people needing attendance, and handling the alert in time. The record of attendance will be stored on the cloud computing platform to analyse the quality of attendance. 


\subsection{Cloud Computing Platform}

The cloud computing platform in the system is responsible for communicating with the mobile device, and its main features are shown in Figure 2.

We establish a personal health information service. The aged or disabled people submit the form on their mobile device to upload information to the platform. Then, the operations like user registration, binding equipment and associating with the relatives and paramedics are completed. The information can be viewed, edited or deleted on the website.

The platform receives alarm information from mobile device, and use database to store massive amounts of healthcare data. Data are displayed through the web page.

Provided with a remote service platform, paramedics and relatives use mobile devices to access the web interfaces, and get alarm information and the corresponding alarm status. Paramedics can make timely care, and relatives may use these results as criteria for assessing the quality of care service.

\section{Architecture Design}

\subsection{Non-Contact Sensors}

Figure 3 shows the schematic of the non-contact sensor, which consists of two parallel copper wires positioned at the bottom of the diaper. Resulting from low cost, the sensor is disposable that it can be discarded with the used diaper to ensure the hygiene security of the aged or disabled people. The value of sensor resistance fell sharply after the urinary incontinence attributing to the big difference between the electric conductivity of dry and wet diapers. Therefore, reliable detection of urinary incontinence is implemented.

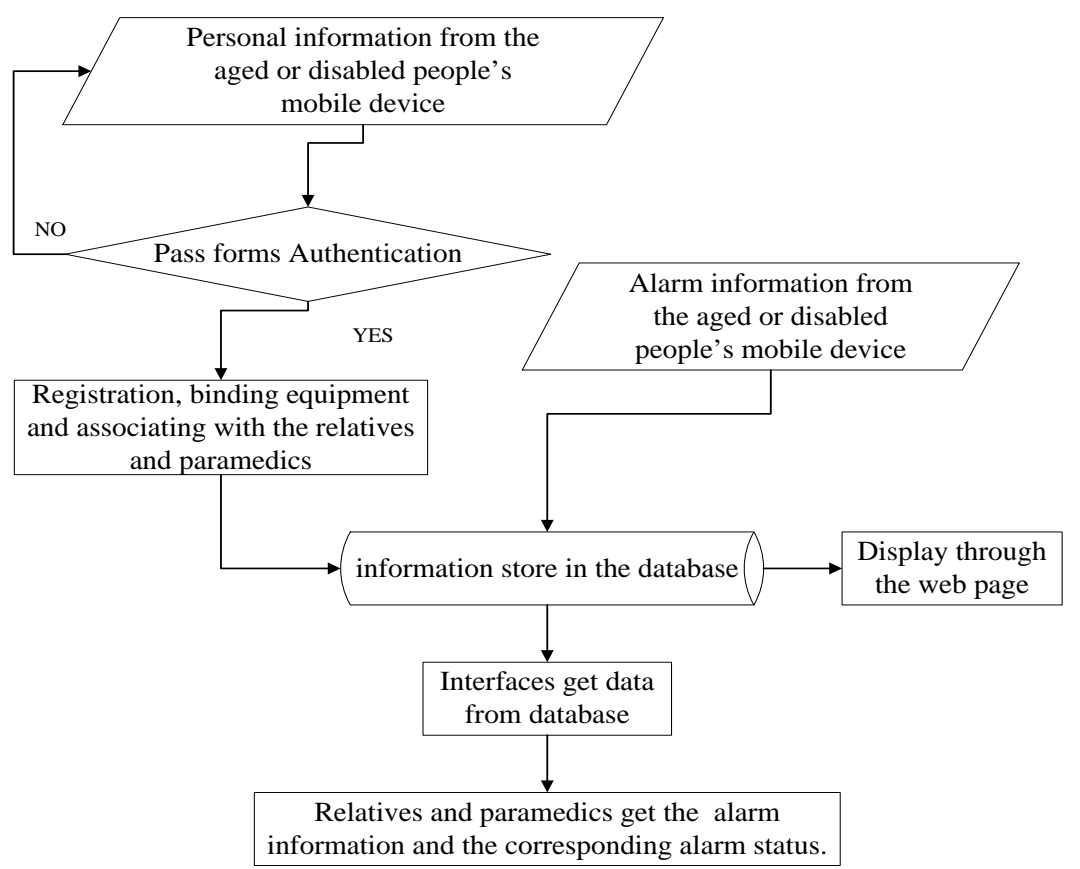

Figure 2. Logic diagram of cloud platform. 


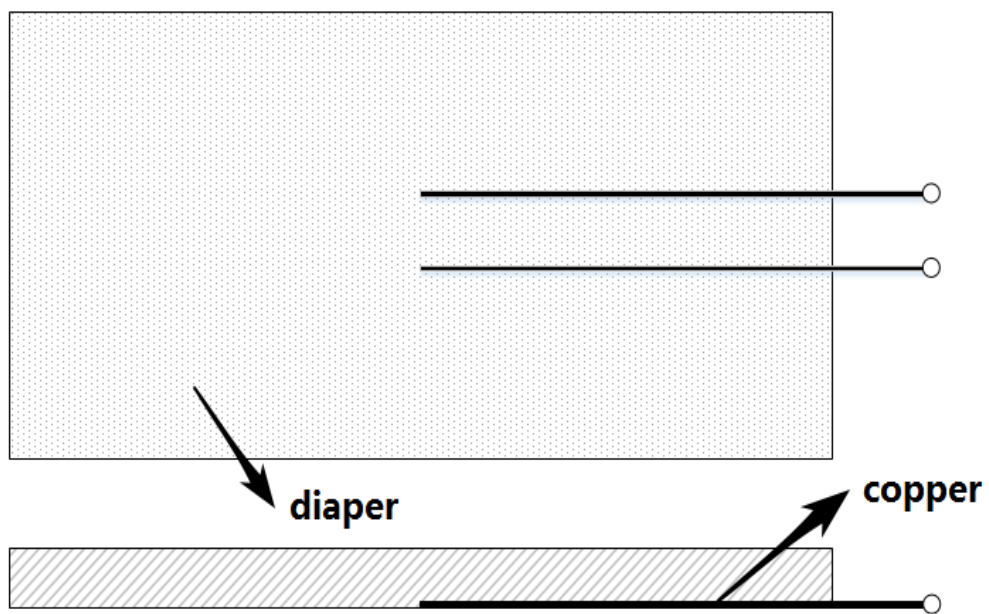

Figure 3. Schematic of the non-contact sensor.

\subsection{Acquisition and Transmission Device}

The device placed at the bedside of aged or disabled people connects to a sensor with a wire, so that there is no special limitation on the volume. While urinary incontinence monitoring and alarm information transmission functions are implemented, an alarm button is added to respond to emergency situations. In addition, a bright LED lamp module is used for night lighting to improve equipment availability. Figure 4 shows the hardware and software block diagrams of the device.

\subsection{Mobile Device Software}

APP development for mobile devices of the system is based on Android equipment, designed in JAVA language, using the MVC framework, requiring android version of at least 4.2.2.

As to the client software for aged or disabled people, it is responsible for patient information management, real-time monitoring diapers wetness state and hardware state. The APP can communicate with hardware devices, back-end server, paramedics' and their relatives' phones. Communicating with hardware devices via Bluetooth 2.0, humidity, battery level and other information from hardware can be obtained in real time. After the hardware de-vice sends a wet alarm, APP will push requests to the server, and the corresponding alarm information will be sent to all paramedics APP, as well as a text message to relatives. At the same time, the alarm information will be stored in log form in the back-end server.

As to the client software for paramedic, it is responsible for tasking paramedic, informing them patients' information, monitoring and recording their nursing situation. After aged or disabled people client APP pushes wet alarm information to paramedic client software through push server, each paramedic can choose to click the alarm message, which is considered as answering to nursing aged or disabled people. Only one paramedic can get the permit, and after an alarm message is answered, it is shown inoperable in other paramedic client 

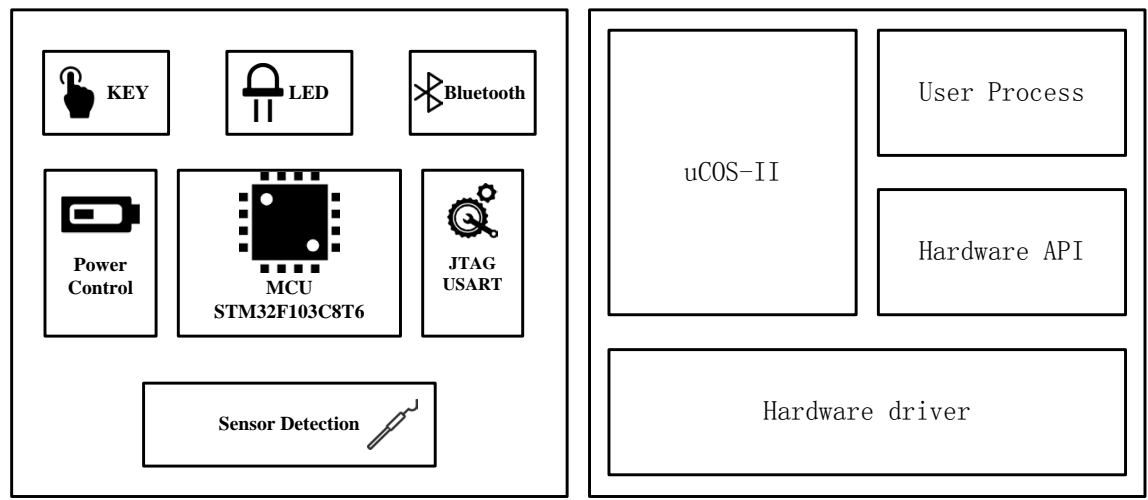

Figure 4. Hardware and software block diagrams.

APP. The alarm message needs to be clicked again when the nursing is finished. All the operations of paramedic will be up-loaded to the back-end server so that the corresponding nursing state can be monitored in real time.

\subsection{Cloud Computing Platform}

In our system, using a web server and a push server to complete the required functions will get a result. The framework diagram of the web and push server is shown in Figure 5.

The web server is responsible for maintaining all user information, alarm state, and history of alarm handling results. Also, it will display the data to the medical staff through web page. The development environment of the web server is based on WAMP (Apache + Mysq1/MariaDB + Perl/PHP/Python under Windows), and the server uses MVC framework, which separates the input, processing procedure and output, so that it eases the management and development procedure. Web server communicates with mobile device via http protocol. The format of information transfer between the mobile device and the server is a JSON string.

Push Server is based on the JPush development platform. The server is small in size, simple in function, and designed in the JAVA language. The data sent by push server are alarm information, and the format is JSON string.

\subsection{Wireless Communication Protocol}

Communication network of the entire system is consisted of the alert monitoring layer and the alert management layer, completing the transmission of alarm information with the help of these two layers. Particularly, two-way communication protocol was designed in two layers respectively, in order to ensure the reliability of transmitting alarm information.

Alarm monitoring layer applied to communications between the mobile device and the acquisition and transmission device. In order to achieve functions like device authentication, device configuration and alarm transmission, the data transmission protocols are rigorous and practically designed. First of all, validation is required when the first connection occurs. As the Figure 6 shows, the 

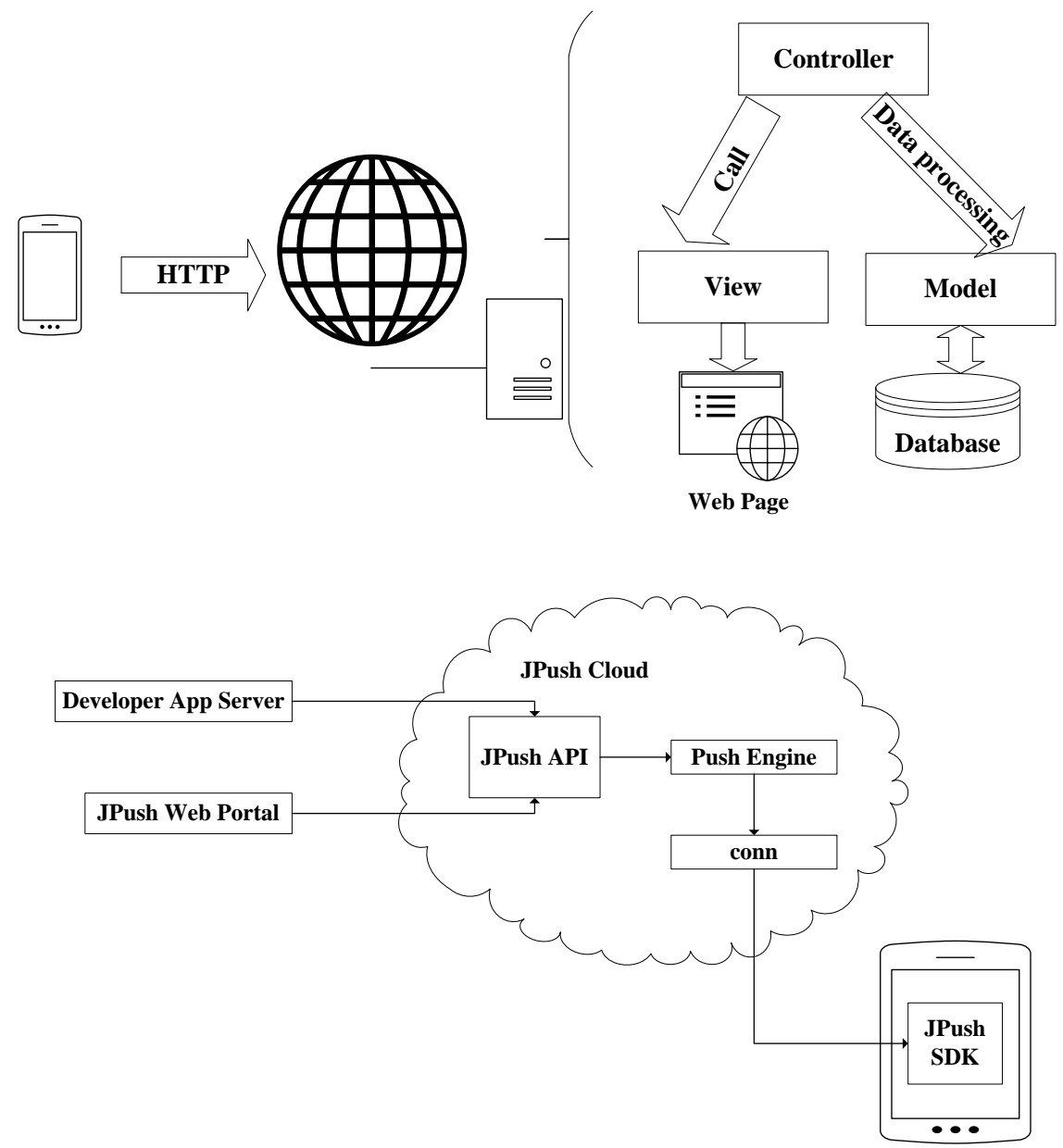

Figure 5. Framework diagram of web and push server.

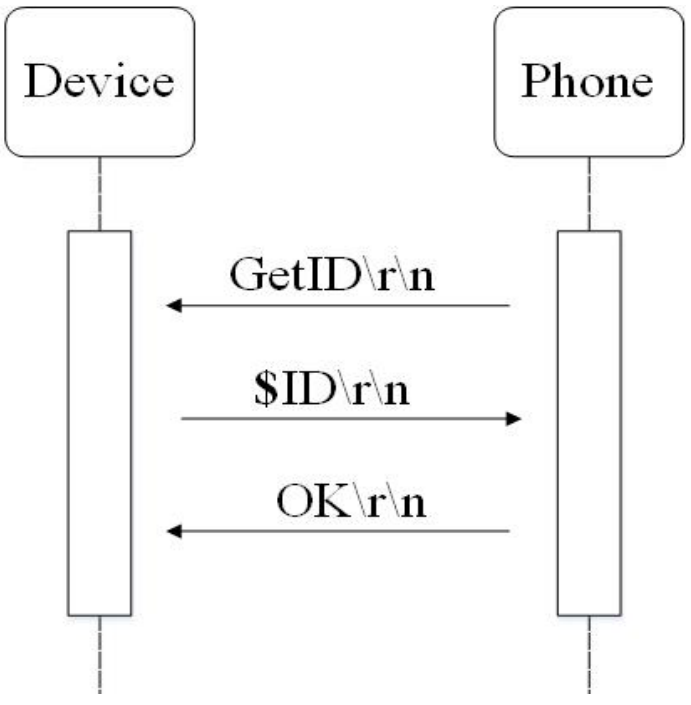

Figure 6. Validation process in alarm monitoring layer.

mobile device sends a command to get the acquisition and trans-mission device's ID, and return confirmation message after it obtains the ID. Secondly, when the connection is established, the mobile device sends the time configura- 
tion command to the acquisition and transmission device, and transmits time after receiving the confirmation message. When the acquisition and transmission device receives the time sent by a mobile device, it will return the confirmation message again, which is illustrated in Figure 7. Thirdly, as shown in Figure 8, when the device detects the change of humidity, alarm information and the time of occurrence will be sent to the mobile device. After receiving the confirmation message from the mobile device, it will send battery level information to the mobile device, and the mobile device will still return message as confirmation. In addition, since the mobile device is based on an embedded operating system, its processing capacity is limited by the microprocessor computing performance. So, there will appear packet loss during the communication process. Based on this challenge, the Bluetooth communication processes of our device were designed robustly, so that the alarm information can be sent out steadily and rapidly.

Alarm managing layer is applied to communications between the mobile de-

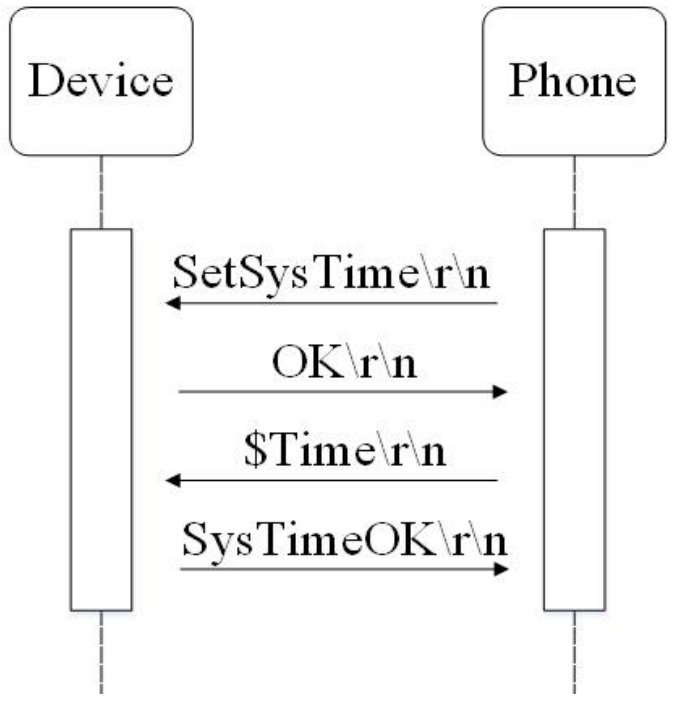

Figure 7. Time configuration in alarm monitoring layer.

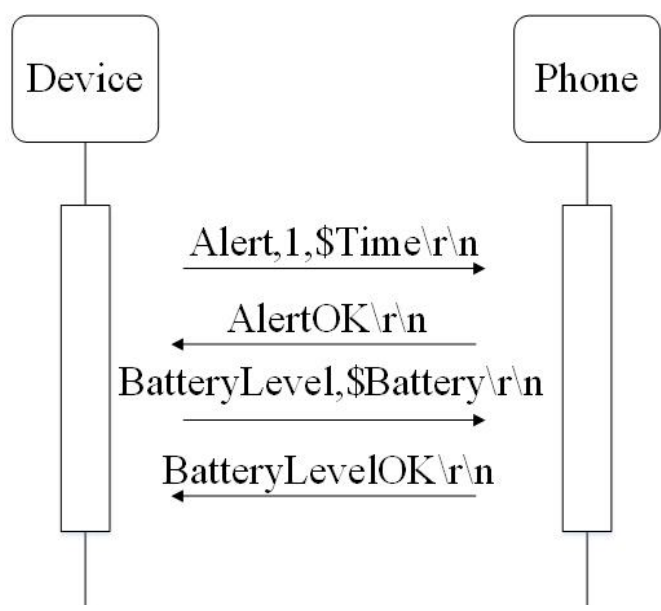

Figure 8. Alert process in alarm monitoring layer. 
vice and the cloud computing platform, processing user registration and alarm information uploading. The server will feedback confirmation of success or failure to the mobile phone after receiving registration data or alarm messages from the phone, as is shown in Figure 9 and Figure 10.

\section{Implementation and Result}

The whole system, from sensor to cloud platform, is accomplished. Figure 11 shows an overview of the system. And the detection of the sensor and the transmission delay of alarm are tested to ensure reliability of the system.

\subsection{The Detection of Sensor}

Quantitative saline is dripped to four specific regions in diaper to simulate the actual situation of urinary incontinence. The alarm threshold of the sensor is set to $10 \mathrm{M}$ ohm and the sensor connects to the acquisition and transmission device during the test. The embedded software specialized in test is developed so the information about resistance and alarm can be received and stored in a mobile device. Figure 12 shows the regions of the diaper in the test and Table 1 shows

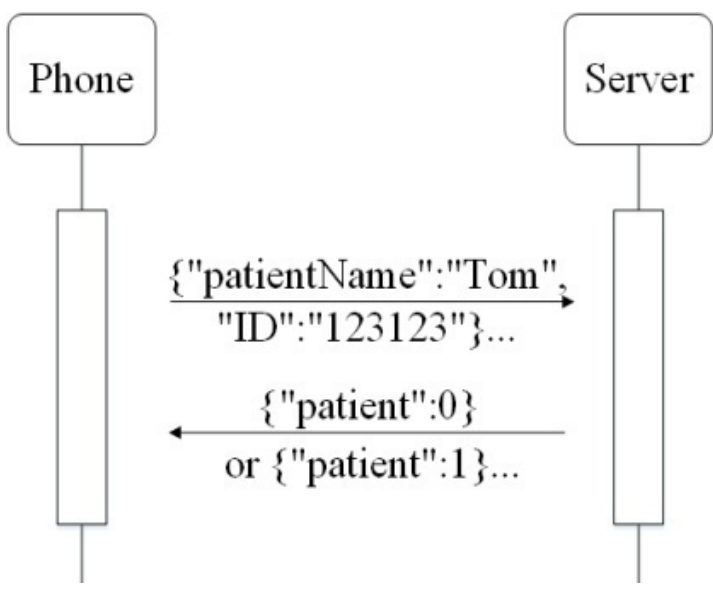

Figure 9. Registration process in alarm managing layer.

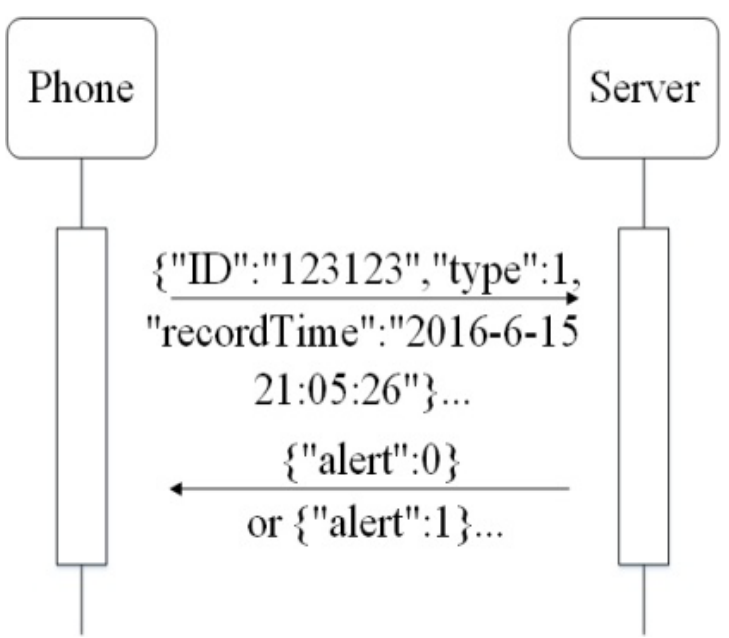

Figure 10. Alert process in alarm managing layer. 


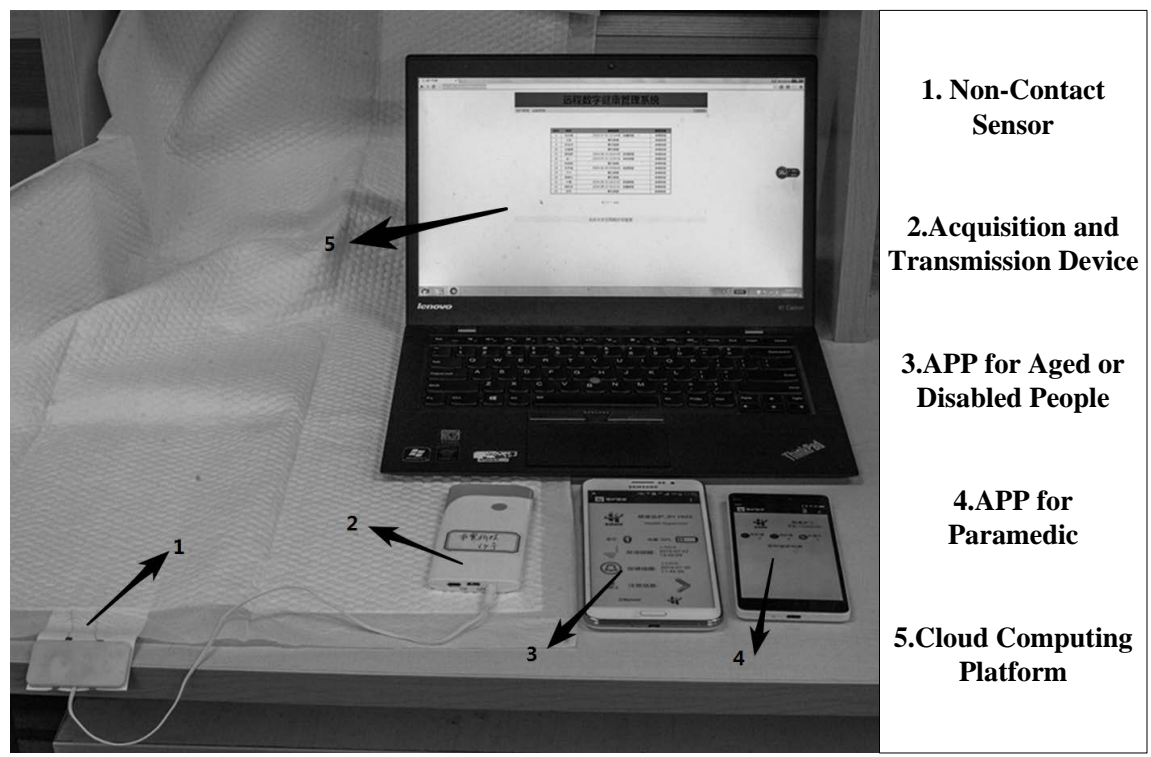

Figure 11. Overview of the monitor system.

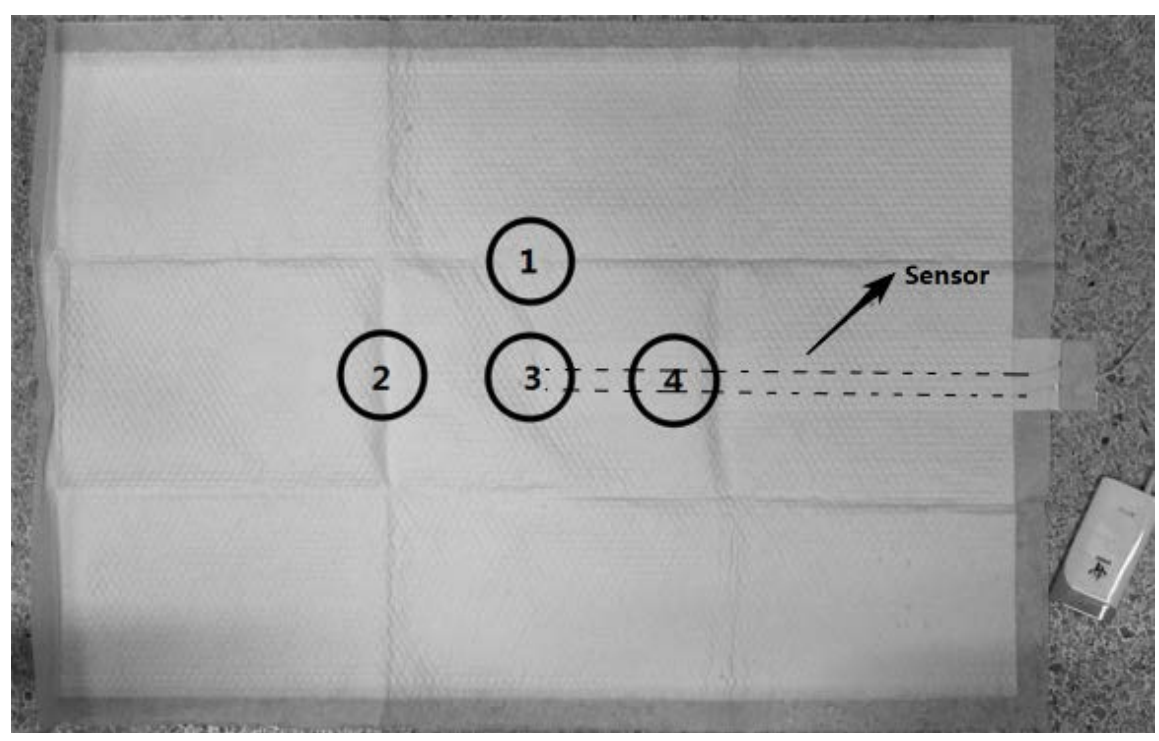

Figure 12. Regions of the diaper in test.

Table 1. Result of sensor test.

\begin{tabular}{cccc}
\hline Region & Saline $(\mathrm{ml})$ & Res $(\mathrm{ohm})$ & Alarm \\
\hline 1 & 50 & $10 \mathrm{M}+$ & $\mathrm{N}$ \\
1 & 100 & $5 \mathrm{M}$ & $\mathrm{Y}$ \\
2 & 50 & $10 \mathrm{M}+$ & $\mathrm{N}$ \\
2 & 100 & $2.3 \mathrm{M}$ & $\mathrm{Y}$ \\
3 & 50 & $5.5 \mathrm{M}$ & $\mathrm{Y}$ \\
3 & 100 & $0.8 \mathrm{M}$ & $\mathrm{Y}$ \\
4 & 50 & $2.7 \mathrm{M}$ & $\mathrm{Y}$ \\
4 & 100 & $0.38 \mathrm{M}$ & $\mathrm{Y}$ \\
\hline
\end{tabular}


the result of the test. Figure 13 shows the resistance change of the sensor during the experiment. When the diaper is dry, the value of resistance is rather large-on the order of 9 . After the drop of saline, the value rapid decline to the order of 5 and finally be stabilized at $0.38 \mathrm{M}$ ohm.

The results show in Table 1, 2 of 8 don't trigger the alarm, during which the saline can't infiltrate to the area where lay the copper wire. In the other experiment the saline reach and wet the sensor so the value goes down rapidly under 6 $\mathrm{M}$, which is less than the threshold value set as $10 \mathrm{M} \mathrm{ohm}$.

\subsection{Transmission Delay of Alarm}

The transmission delay of alarm is tested by sending the manual alarm to mobile device. Sending message and uploading alarm to cloud platform via android operating system is reliable, and the delay is decided by network status. Five testers are invited and complete 282 sending alarm tests. All the manual alarms are received by two apps, relatives and cloud platform. The time delay is recorded and shown in Figure 14. Most of the time delay is less than 30 seconds, while the longest time delay reaches to almost 100 seconds.

\section{Conclusion}

We have presented a real time wireless monitor system for urinary incontinence, which is applied in two nursing homes in Beijing and Shanghai as probation. The system is designed, reliable and rapid response to emergency situations. The

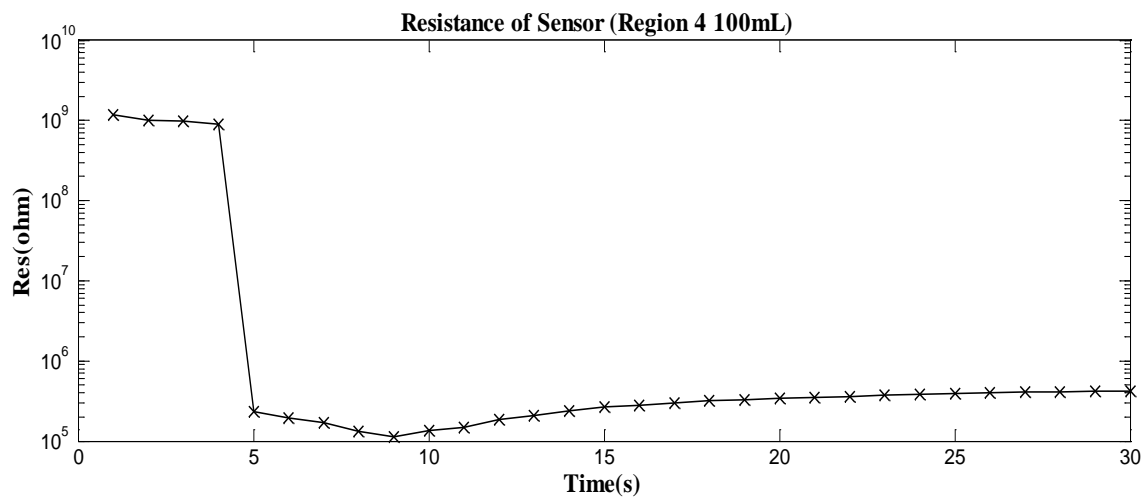

Figure 13. Regions of the diaper in test.

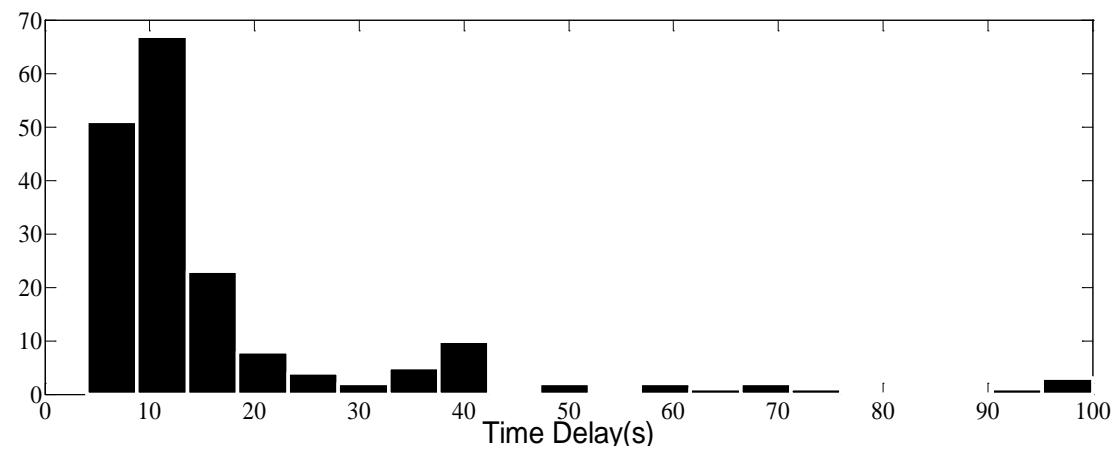

Figure 14. Distribute of time delay. 
system can detect urinary incontinence accurately and transfer the alarm information reliably owing to the self-design sensor and bidirectional transmission protocol. The application of cloud platform makes the system can record all the alarm information and handle emergency rapidly. There still exist some limitations such as the time delay is too long in sometimes and the mobile device apps are just supported by an android.

\section{Acknowledgements}

The authors gratefully acknowledge support from School of Electronics Engineering and Computer Science, Peking University.

\section{References}

[1] Zheng, W., Lin, S.J. and Chen, K. (2014) Characteristics and Trend of Population Aging in China and Its Potential Impact on Economic Growth. Journal of Quantitative \& Technical Economics, 8, 3-20.

[2] Li, K., Du, Y.Q. and Wang, Q.Z. (2014) Design of a New Kind of Non-Contact Wet Monitoring Device. Microcomputer \& Its Applications, 7, 87-90. https://doi.org/10.1007/978-3-540-69916-3 8

[3] Biswas, J., et al. (2008) Design of a Smart Continence Management System Based on Initial User Requirement Assessment. Smart Homes and Health Telematics, International Conference, Ames, July 2008, 62-72. http://doi.org/10.1007/978-3-540-69916-3 8

[4] Xing, Y.Y. (2013) Diapers Device of Microcontroller Phone Alarm Which Is Based on the STC90C52RC Microcontroller. Electronics Quality, 3, 44-54. https://doi.org/10.1109/ECTICON.2008.4600374

[5] Ang, L.M., et al. (2008) Wireless Intelligent Incontinence Management System Using Smart Diapers. International Conference on Electrical Engineering/ Electronics, Computer, Telecommunications and Information Technology, Krabi, May 2008, 69-72. http://doi.org/10.1109/ECTICON.2008.4600374

\section{Scientific Research Publishing}

Submit or recommend next manuscript to SCIRP and we will provide best service for you:

Accepting pre-submission inquiries through Email, Facebook, LinkedIn, Twitter, etc. A wide selection of journals (inclusive of 9 subjects, more than 200 journals)

Providing 24-hour high-quality service

User-friendly online submission system

Fair and swift peer-review system

Efficient typesetting and proofreading procedure

Display of the result of downloads and visits, as well as the number of cited articles

Maximum dissemination of your research work

Submit your manuscript at: http://papersubmission.scirp.org/

Orcontactijcns@scirp.org 